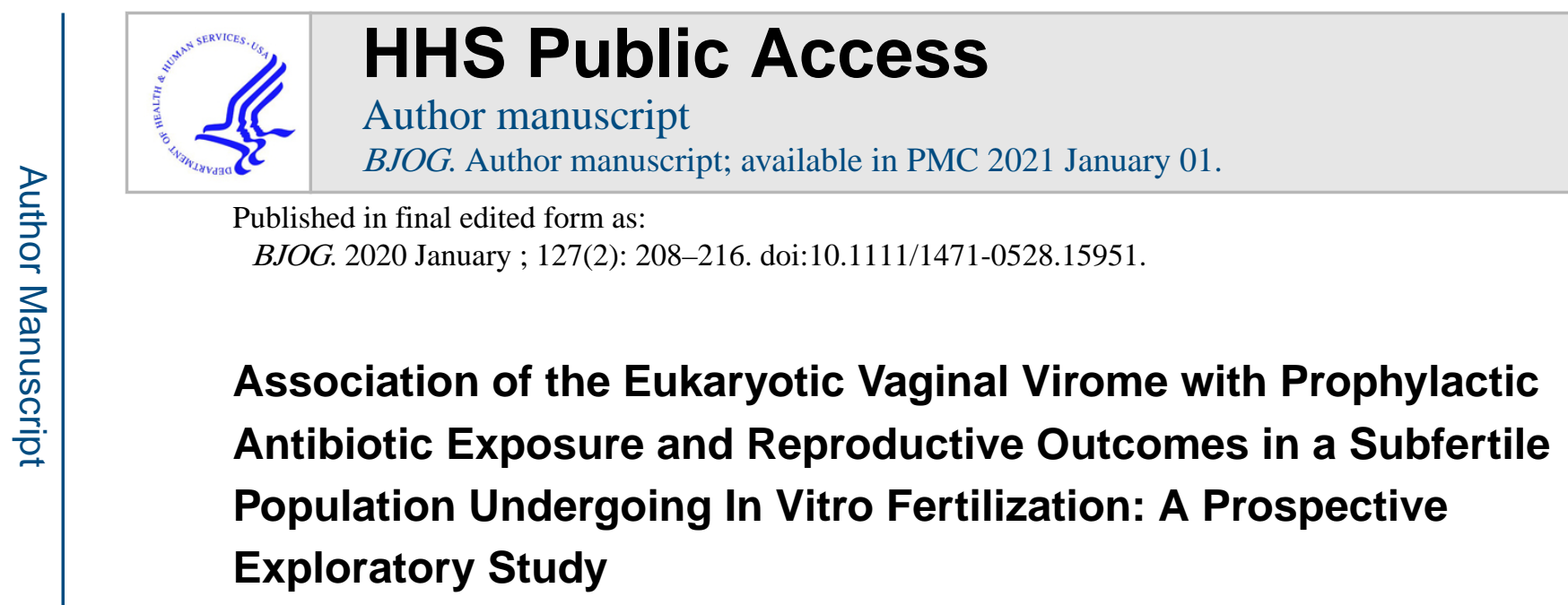

\author{
Ashley M. Eskew ${ }^{1}$, Molly J. Stout ${ }^{2}$, Bronwyn S. Bedrick, Joan K. Riley ${ }^{1,1}$, Kenan R. \\ Omurtag $^{1}$, Patricia T. Jimenez ${ }^{1}$, Randall R. Odem ${ }^{1}$, Valerie S. Ratts ${ }^{1}$, Sarah L. Keller ${ }^{1}$, Emily \\ S. Jungheim ${ }^{1}$, Kristine M. Wylie ${ }^{3,4}$ \\ ${ }^{1}$ Washington University in St. Louis, Department of OB-GYN, Division of Reproductive \\ Endocrinology and Infertility, 4444 Forest Park Ave., Suite 3100, St. Louis, MO 63108 \\ ${ }^{2}$ Washington University in St. Louis, Department of OB-GYN, Division of Maternal Fetal Medicine, \\ 4901 Forest Park Ave, Suite 10134, St. Louis, MO 63108 \\ ${ }^{3}$ Washington University in St. Louis, Department of Pediatrics, Division of Infectious Diseases, \\ 660 S. Euclid Ave, Box 8208, St. Louis, MO 63110 \\ ${ }^{4}$ Washington University in St. Louis, McDonnell Genome Institute, 4444 Forest Park Ave, St. \\ Louis, MO 63108
}

\begin{abstract}
Objective: The objective of this study was to use high-throughput sequencing to describe the vaginal eukaryotic DNA virome in patients undergoing in vitro fertilization (IVF) to examine associations between the vaginal virome, antibiotic exposure, and IVF outcomes.
\end{abstract}

Design: Prospective exploratory study

Setting: Single Academic Fertility Center

Population: Subfertile women age 18-43 undergoing their first IVF cycle with a fresh embryo transfer.

\footnotetext{
Corresponding Author: Kristine Wylie, PhD, 660 S. Euclid Avenue, Box 8208, St. Louis, MO 63110, 314-747-4069, kwylie@wustl.edu. Contribution to Authorship:

AME, MJS, BSB, ESJ and KMW made substantial contributions to the conception and design of the study, acquisition, analysis and interpretation of the data for the work, drafting the manuscript, revising it critically, final approval of the version to be published and agrees to be accountable for all aspects of the work.

JKR, PTJ, VSR, KRO, SLK and RRO made substantial contributions to the analysis and interpretation of the data for the work, revised the manuscript critically, gave final approval of the version to be published and agrees to be accountable for all aspects of the work.

Details of Ethics Approval:

This is a prospective exploratory study conducted within an ongoing randomised control non-inferiority trial at a single academic institution. This planned exploratory analysis as well as the parent non-inferiority randomised trial was approved by Washington University's in St. Louis, MO Institutional Review Board (IRB ID\# 201709018) on January 24, 2018 and was registered at clinicaltrials.gov ( prior to enrollment of the first study participant.
} 
Methods: The primary exposure was prophylactic azithromycin or no azithromycin prior to IVF. Patients had a mid-vaginal swab obtained at the time of embryo transfer for virome analysis.

Main Outcome Measures: The primary outcome compared between exposure groups was characteristics of vaginal virome and clinical pregnancy rates. Secondary outcomes were virome associations with number of oocytes retrieved, number of blastocysts, and implantation rate.

Results: Twenty-six subjects contributed a vaginal swab prior to embryo transfer. There were no significant differences in IVF outcomes between azithromycin groups. There was no association between viral diversity and clinical pregnancy overall. A higher diversity of herpesviruses and alphapapillomaviruses were observed in samples from the azithromycin-treated group compared to the no azithromycin group ( $\mathrm{p}=0.04)$. In subjects that received azithromycin, viral diversity was higher in the group that did not achieve clinical pregnancy compared to those who did $(\mathrm{p}=0.06)$.

Conclusions: We demonstrate the vaginal eukaryotic virome in women undergoing IVF is associated with antibiotic exposure. Additionally, we demonstrate an inverse trend between viral diversity and pregnancy, with a higher number of viruses detected associated with failure to achieve clinical pregnancy in the azithromycin group.

\section{Tweetable Abstract}

Higher viral diversity is associated with prophylactic antibiotic exposure in subfertile women undergoing IVF.

\section{Keywords}

in vitro fertilization; vaginal virus; virome; embryo transfer; reproductive tract microbiome

\section{INTRODUCTION}

The most recent Centers for Disease Control National Survey of Family Growth estimates that 1.5 million married women between ages 15 and 44 years are infertile. ${ }^{1}$ The 2017 Society for Assisted Reproductive Technology summary report indicates that success rate per egg retrieval (judged as live birth) is far from optimized at $46.8 \%$ for women $<35$ years. Success drops precipitously with increasing age to $10.1 \%$ at age $41-42$ and $3.1 \%>42$ years. 2

Research continues to identify factors that affect success rates of in vitro fertilization (IVF). Recent evidence suggests the bacterial community composition in the reproductive tract may influence IVF outcomes. A Lactobacillus-dominated endometrium and lower vaginal bacterial diversity have been associated with higher implantation and live birth rates after IVF. $^{3-5}$

Bacterial communities alone, however, do not fully represent the vaginal microbiome, as other microbes, including viruses, are also present in the reproductive tract and have clinical implications. Viruses within human microbial communities, "the human virome," constitute an important but understudied component of the microbiome. In particular, eukaryotic viruses, which can directly infect human cells are of interest as they can cause symptomatic and subclinical infections that impact health. For example, in the Human Microbiome 
Project, vaginal samples from asymptomatic, healthy women contained viruses that could cause symptomatic disease, including herpesvirus and oncogenic papillomaviruses. ${ }^{6,7}$ Additionally, higher vaginal eukaryotic viral diversity (the number of viruses detected) has been associated with preterm birth in an obstetric population. ${ }^{8,9}$ These findings suggest that vaginal eukaryotic viruses may influence reproductive outcomes. The vaginal virome has yet to be described in a preconception cohort.

One common step in IVF protocols is to prophylactically administer antibiotics to reduce the theoretic risk of infecting the endometrial cavity during embryo transfer. However, little evidence supports the clinical benefit of this practice, and its effect on the vaginal microbiome is unclear. ${ }^{10-13}$ Recent studies have shown that antibiotics can have unexpected effects on virus replication and pathogenesis, suggesting the virome may be affected by antibiotic administration as well. ${ }^{14,15,16,17}$

We hypothesize that the vaginal virome is associated with IVF clinical outcomes and may be influenced by prophylactic antibiotic exposure. The objective of the present study is to: 1) describe the vaginal virome at the time of embryo transfer via high-throughput sequencing 2) explore the potential impact of prophylactic azithromycin on the vaginal virome and 3) examine the association between the vaginal virome and IVF outcomes.

\section{METHODS}

This is a planned a priori prospective exploratory study conducted within an ongoing randomised control non-inferiority trial at a single academic institution. This planned exploratory analysis as well as the parent non-inferiority randomised trial was approved by Washington University's Institutional Review Board (IRB ID\# 201709018) on January 24, 2018 and the parent trial was registered at clinicaltrials.gov () prior to enrollment of the first study participant. Written informed consent was obtained from each subject prior to enrollment. The ongoing parent randomised controlled trial was designed to examine the effects of prophylactic azithromycin versus no azithromycin on the outcome of clinical pregnancy following IVF. For this planned nested prospective cohort, we leveraged the randomization of patients undergoing IVF to azithromycin or no azithromycin to examine the effect of azithromycin on viral communities in the vagina. The analysis of this cohort study was performed according to STROBE guidelines ${ }^{18}$. The STROBE flow diagram for the study appears online as Figure S1.

Inclusion criteria for this exploratory study were the female partner must be aged 18-43 years and undergoing her first fresh IVF cycle. Exclusion criteria were: any contraindication to antibiotic treatment, not intending to undergo fresh embryo transfer (e.g., fertility preservation patients and oocyte donors), failure to have an embryo transfer, use of extended antibiotic coverage at time of egg retrieval, already taking antibiotics for any reason (e.g., upper respiratory infection), history of pelvic infection, use of donor or frozen sperm, or planned limited insemination (inseminating a limited number of the oocytes retrieved). Subjects for this exploratory study were enrolled from February 2018 to September 2018. 
Randomization to prophylactic azithromycin or no azithromycin in the parent trial occurred in 1:1 ratio and was performed in a block-randomised fashion via computer random number generator. The azithromycin group received the current standard regimen in our clinic: 1gram azithromycin oral once for both partners the day controlled ovarian stimulation is initiated with injectable gonadotropins. The no azithromycin treatment group received no azithromycin for either partner. The remainder of the IVF protocol and ovarian stimulation was at the discretion of the treating reproductive endocrinologist. All subjects had 2-g cefazolin administered immediately following oocyte retrieval, which is standard surgical prophylaxis in our center. The primary exposure was azithromycin exposure. The primary outcomes were vaginal virome composition and clinical pregnancy. For this planned exploratory study, a convenience sample size of 32 was estimated to achieve a goal of at least 12 patients in each antibiotic group after accounting for loss to follow up and sample failures.

To sample vaginal virome communities mid-vaginal swabs were obtained immediately prior to embryo transfer. A sterile speculum was inserted into the vaginal canal and a sterile dualtipped rayon swab (Starplex Scientific, Ontario, Canada) was applied 3-5 times to both lateral sidewalls of the vaginal canal. Each swab was placed immediately into a sterile collection tube and stored at $-80^{\circ} \mathrm{C}$ until batch analysis. Embryologists were blinded to subject treatment group allocation.

DNA was extracted using the QIAmp BiOstic Bacteremia DNA Kit (Qiagen, Germantown, Maryland). Dual-indexed sequencing libraries were constructed with the IDT Lotus DNA Library kit (Integrated DNA Technologies, Coralville, Iowa), and viral DNA was enriched with our custom ViroCap targeted sequencing capture probes that target and enrich complete genomes from all vertebrate viruses (synthesized by Roche NimblelGen, Madison, Wisconson). ${ }^{19}$ Sequences were generated on the Illumina NovaSeq instrument (Illumina, San Diego, CA). Sequences were analyzed based on nucleotide and translated amino acid similarity to reference genomes as previously described. ${ }^{8}$

Baseline and demographic characteristics were compared between those who received azithromycin versus no azithromycin using parametric and nonparametric tests as appropriate. Vaginal viral and microbial community characteristics were compared between those who received azithromycin and those who did not. We then tested for associations between the vaginal virome and clinical pregnancy rate (defined by gestational sac with crown rump length and cardiac activity on ultrasound). Secondary clinical outcomes assessed included: number oocytes recovered, fertilization rate, total number blastocysts (sum of blastocysts transferred or frozen), implantation rate (number of gestational sacs/ number of embryos transferred) and miscarriage rate (clinical loss prior to 20 weeks' gestation).

All analyses were performed in R (www.r-project.org), STATA 12.0 (College Station, TX), SPSS 24 (IBM, Armonk, NY), and GraphPad Prism (La Jolla, CA) statistical programs. R statistical programming language was used to plot data and carry out statistical tests to compare viral communities between and within groups. The Wilcoxon rank-sum was used to compare diversity measures between and within the two groups. Fisher's exact test was used 
to compare the prevalence of viruses between and within the two groups. Spearman correlations were used to test overall associations between viral diversity and clinical features. Hodges-Lehmann (HL) estimate of difference was used to determine 95\% confidence intervals (CI).

This work was funded by T32HD055172 from the National Institute of Child Health and Human Development of the National Institutes of Health; the Washington University Institute of Clinical and Translational Sciences which is, in part, supported by the NIH/ National Center for Advancing Translational Sciences (NCATS), CTSA grant UL1TR002345; and by the Children's Discovery Institute of Washington University and St. Louis Children's Hospital. The study was not supported by a peer reviewed grant and did not undergo priority assessment. The funders had no role in conducting the research or writing the paper.

\section{RESULTS}

A total of 32 subjects were enrolled. Of these, four became ineligible after randomization (two decided to do preimplantation genetic testing, one never started their stimulation medications, one was undergoing her second IVF cycle) and two were excluded after randomization due to failure to have an embryo transfer (one subject had their cycle cancelled due to poor response and one subject had failed fertilization). Twenty-six subjects contributed 26 swabs for analysis.

Baseline characteristics were compared between groups. AMH was significantly higher in the no azithromycin group compared to the prophylactic azithromycin group $(\mathrm{p}<0.05)$. Accordingly, there was a significant difference in the types of stimulation protocols used between groups, with $33.3 \%$ of the azithromycin group using a flare protocol (poor responder protocol) versus $0 \%$ in the no azithromycin group ( $\mathrm{p}=0.03$ ) (Table 1). Other baseline and demographic variables did not differ between azithromycin groups (Table 1). There were no significant differences in cycle level variables between the two groups including peak estradiol or total gonadotropins used (Table S1). There were no significant differences in IVF clinical outcomes between subjects who received prophylactic azithromycin at baseline and those who did not (Table S2).

We detected at least one virus in 22 out of 26 subjects. We detected four families of viruses present in the vagina at the time of embryo transfer: Herpesviridae, Polyomaviridae,

Papillomaviridae, and Anelloviridae, with Papillomaviridae being the most common (Figure 1). Notably, only one type of papillomavirus covered in the nine-valent human papillomavirus vaccine was detected in one patient out of the entire cohort. All four subjects with no viruses detected had a positive clinical pregnancy. There was no apparent difference in the types of viruses present in those who achieved clinical pregnancy versus those who did not or in the prophylactic azithromycin versus no azithromycin groups (Figure 1).

We noted a modest association between a lower viral diversity at the time of embryo transfer and clinical pregnancy, however, this did not reach statistical significance ( $\mathrm{p}=0.3, \mathrm{HL}-1$; 95\% CI, -2 to 1) (Figure 2A). When we looked only at herpesviruses and 
alphapapillomaviruses, those most likely to result in clinical phenotypes in the reproductive tract, we found no association between viral diversity and clinical pregnancy $(\mathrm{p}=0.6$, HL 0 ; 95\% CI, -1 to 1) (Figure 2B). There was no statistical difference between the number of viruses detected in the prophylactic azithromycin versus no azithromycin groups $(\mathrm{p}=0.9, \mathrm{HL}$ 0; 95\% CI, -1 to 2) (Figure 2C); however, we did find that more herpesviruses and alphapapillomaviruses were observed in the azithromycin-treated group ( $\mathrm{p}=0.04$, HL 1; 95\% CI, 0 to 1) (Figure 2D).

Viral diversity and cycle level variables were then compared between azithromycin randomization groups. In subgroup analysis amongst subjects who received prophylactic azithromycin, there was no significant differences in terms of age, BMI, antral follicle count (AFC) or duration of infertility between those with and without a clinical pregnancy. However, in the group that did not become pregnant, AMH was significantly lower $(\mathrm{p}=0.03$, HL $1.8 ; 95 \%$ CI 0.2 to 4) (Figure 3A) than in the group that became pregnant. Furthermore, $40 \%$ of subjects had a diagnosis of diminished ovarian reserve versus $0 \%$ of women who were clinically pregnant. There were no significant differences in potential confounders when comparing the subjects that did not get pregnant between prophylactic azithromycin versus no azithromycin groups including age, BMI, AFC or duration of infertility. However, $60 \%$ underwent a flare cycle in the azithromycin arm, compared to $0 \%$ in the no azithromycin group.

In the group that received prophylactic azithromycin, peak estradiol levels were lower ( $\mathrm{p}=0.02$, HL 931; 95\% CI 65 to 1843) (Figure 3B) and viral diversity was higher in the group that did not achieve clinical pregnancy compared to those who became pregnant ( $\mathrm{p}=0.06, \mathrm{HL}-2 ; 95 \% \mathrm{CI}-3$ to 0 ) (Figure 3C). The numbers of herpesviruses and alphapapillomaviruses in each sample trended higher in the group that received prophylactic azithromycin and did not achieve clinical pregnancy compared to those who became pregnant, but the difference was not statistically significant ( $\mathrm{p}=0.5$, HL $0 ; 95 \% \mathrm{CI},-1$ to 2 ) (Figure 3D).

Viral diversity was not correlated with AFC, AMH, total amount of gonadotropin, peak estradiol, number of oocytes retrieved, number of mature oocytes, number of blastocysts, or implantation rate (Table S3). Only one subject had a miscarriage after a documented clinical pregnancy and did not have any unique virome characteristics, therefore, we were unable to make meaningful conclusions for this outcome.

\section{DISCUSSION}

\section{Main Findings:}

We demonstrate that eukaryotic, DNA viruses are found in vaginal swabs from asymptomatic subfertile women at the time of embryo transfer. A higher number of viruses trended toward an association with a lower likelihood of clinical pregnancy and all four subjects that had no viruses detected had a positive pregnancy outcome; although these findings did not reach statistical significance, they are worthy of exploration in a larger cohort. The number of viruses detected did not associate with secondary clinical endpoints. 
Administration of prophylactic azithromycin did not associate with the total number of viruses. However, it associated with a higher number of herpesviruses and alphapapillomaviruses. Also, the number of viruses detected was higher in the group of women that did not achieve clinical pregnancy in the prophylactic azithromycin group compared to those who did. This group of women also had lower estradiol levels, which was likely attributable to infertility diagnosis and baseline AMH. As a result, this group underwent more flare (poor responder) protocols. Women who underwent a flare protocol and did not achieve pregnancy tended to cluster together with a higher number of viruses detected, however, the numbers were too small to make meaningful conclusions.

\section{Strengths and Limitations:}

Strengths of our study include its novel findings and prospective design. This is the first study to our knowledge to describe the vaginal virome in the immediate preconception period and to explore the impact of antibiotic exposure on the vaginal virome. The use of ViroCap targeted sequence capture enabled comprehensive sequencing of viral communities with a high level of sensitivity. ${ }^{19,20}$ Although AMH and cycle type were significantly different between randomization groups they were not associated with clinical pregnancy and therefore are unlikely to significantly influence our findings.

Despite the above strengths, limitations must be considered. First, due to the descriptive nature of this study, our sample size was small and underpowered to detect differences in clinical endpoints. As this was an exploratory study, we did not adjust p-values to correct for multiple testing, although we detected trends that will serve to generate hypotheses and inform power considerations in future studies.

Second, every subject received cefazolin for surgical prophylaxis at the time of egg retrieval, which is our practice's standard of care. Therefore, the potential confounding effect of cefazolin cannot be ruled out. As antibiotic prophylaxis is indicated at our institution when entering the abdominal cavity through the genitourinary tract, a control group would not be feasible for this study. Additionally, the time between antibiotic exposure and swab collection varied between subjects, ranging from 10 to 17 days for azithromycin and 3 to 5 days for cefazolin, making a temporal relationship between exposure and effect on the vaginal virome difficult to appreciate.

We observed a single time point in the IVF cycle, and while prior studies would suggest that the microbiome at the time of embryo transfer correlates with clinical outcomes, the potential importance of viral community trends throughout the IVF cycle is unknown. ${ }^{3-5}$ Future studies could evaluate virome dynamics longitudinally through preconception and pregnancy, which may reveal either specific time points or dynamic changes over time that associate with clinical outcomes. ${ }^{8}$

A further limitation is that DNA sequencing does not distinguish viral exposure from active replication, which should be considered when interpreting our results. We only assessed eukaryotic DNA viruses, which includes clinically relevant families commonly found in the vagina but does not account for equally important but rarer RNA viruses. To assess viral 
transcription, which is suggestive of active replication of DNA viruses, and to detect RNA viruses, RNA sequencing should be considered for future studies.

Lastly, this was a predominately white, non-Hispanic, subfertile cohort preparing to undergo IVF with a fresh embryo transfer. Therefore, our findings may not be generalizable to a general reproductive population or women undergoing frozen embryo transfers.

\section{Interpretation:}

Recent evidence suggests that a Lactobacillus-dominated endometrium and a vaginal bacterial community with low diversity at the time of embryo transfer have been associated with significantly higher implantation, clinical pregnancy and live birth rates in women undergoing IVF. ${ }^{3-5}$ In our small cohort, we found a trend toward an inverse correlation between viral diversity and clinical pregnancy in the women who received prophylactic azithromycin, suggesting that features of both bacterial and viral components of the vaginal microbiome may associate with clinical pregnancy. However, our small sample size does not allow us to reach a robust conclusion regarding pregnancy outcomes. The association of viral diversity with clinical pregnancy was not observed in the women who did not receive azithromycin suggesting that the association between viral diversity and pregnancy outcome is observed under some conditions, which may include azithromycin exposure. Larger, more expansive studies are needed to understand the biological effects of antibiotics on the viruses in the vagina and implications for clinical outcomes.

Findings from an obstetric population demonstrated that patterns of decreasing eukaryotic viral diversity across trimesters paralleled the dynamic changes of bacterial diversity, suggesting that physiologic changes throughout pregnancy may result in parallel changes within bacterial and viral communities. ${ }^{8}{ }^{9}$ Notably, in that cohort a higher number of viruses detected associated with spontaneous and indicated preterm birth, providing a potential indicator to identify at-risk populations. ${ }^{8}$ The "double hit hypothesis" posits a potential mechanism wherein an asymptomatic maternal viral infection could predispose or sensitize the placental response to bacteria, resulting in pre-term labor. ${ }^{21}$ Taken together, these data suggest that monitoring the vaginal microbiome before and throughout pregnancy may help identify subjects who are at risk for poor IVF and pregnancy outcomes. This knowledge could identify the development of earlier, more effective interventions to improve obstetric outcomes.

Inflammation and infection are considered common links to preterm birth, and while broadspectrum antibiotics are commonly used in management, they have not been shown to prevent preterm birth and in some cases may actually cause harm. ${ }^{22-27}$ Recent evidence has highlighted the unexpected ability of antibiotics to interfere with virus replication and pathogenicity by several mechanisms. ${ }^{14,15,28}$

Azithromycin has demonstrated antiviral activity against Zika virus entry and has the ability to modulate immune response to respiratory syncytial virus (RSV) induced bronchiolitis. 15, 16 Macrolides influence a variety of immunologic response mechanisms resulting in an anti-inflammatory effect, which is central to many early reproductive processes including embryogenesis and implanation. ${ }^{28,29}$ We describe a single dose of azithromycin 10 to 17 
days before embryo transfer, which associated with higher numbers of herpesviruses and alphapapillomaviruses. This suggests the anti-inflammatory effect of azithromycin may make it difficult to contain virus replication or may promote virus reactivation, which may be relevant here. While these studies differ from our study, they highlight potential directions for future research.

\section{CONCLUSIONS}

In conclusion, we demonstrate that eukaryotic viruses are found in vaginal swabs from asymptomatic women in the immediate preconception period. Azithromycin prophylaxis associated with higher diversity of herpesvirus and alphapapillomaviruses. Subjects who did not achieve clinical pregnancy in the group that received prophylactic azithromycin had a greater viral diversity, lower AMH, and lower peak estradiol than those who achieved pregnancy. Future, larger trials should be directed toward assessing the vaginal virome across the IVF cycle to determine the acute impact of prophylactic antibiotics and repeated exposures on the vaginal virome and associations with reproductive outcomes.

\section{Supplementary Material}

Refer to Web version on PubMed Central for supplementary material.

\section{Funding Statement:}

Dr. Eskew's time was supported by the National Institute of Child Health and Human Development of the National Institutes of Health grant number T32HD055172 as a part of the Reproductive Epidemiology Training Program at Washington University in St. Louis. This project was also supported by the Washington University Institute of Clinical and Translational Sciences which is, in part, supported by the NIH/National Center for Advancing Translational Sciences (NCATS), CTSA grant UL1TR002345, and by the Children's Discovery Institute of Washington University and St. Louis Children's Hospital.

Disclosures of interests:

KMW discloses that a patent is pending for ViroCap and receipt of funding support for this study from the Children's Discovery Institute of Washington University and St. Louis Children's Hospital. KMW, AME and ESJ disclose that they have received grant funding for this project from the National Institute of Health and Human Development of the National Institutes of Health, and the National Center for Advancing Translational Sciences.

The authors, MJS, BSB, JKR, KRO, PTJ, RRO, VSR and SLK have no conflicts of interest to disclose.

Completed disclosure of interest forms are available to view online as supporting information.

\section{References:}

1. Chandra A, Copen CE, Stephen EH. Infertility Service Use in the United States: Data from the National Survey of Family Growth, 1982-2010 National Health Statistics Reports; no 73 Hyattsville, MD: National Center for Health Statistics 2014.

2. Society for Assisted Reproductive Technology. c1996- [cited 2019 May 10] Available from: https:// www.sartcorsonline.com/rptCSR_PublicMultYear.aspx?reporting Year=2017.

3. Moreno I, Codoner FM, Vilella F, Valbuena D, Martinez-Blanch JF, Jimenez-Almazan J, et al. Evidence that the endometrial microbiota has an effect on implantation success or failure. Am J Obstet Gynecol. 2016;215(6):684-703. [PubMed: 27717732]

4. Haahr T, Humaidan P, Elbaek HO, Alsbjerg B, Laursen RJ, Rygaard K, et al. Vaginal microbiota and IVF outcomes: development of a simple diagnostic tool to predict patients at risk of a poor reproductive outcome. J Infect Dis. 2018;219(11):1809-17. 
5. Hyman RW, Herndon CN, Jiang H, Palm C, Fukushima M, Bernstein D, et al. The dynamics of the vaginal microbiome during infertility therapy with in vitro fertilization-embryo transfer. J Assist Reprod Genet. 2012;29(2):105-15. [PubMed: 22222853]

6. Peterson J, Garges S, Giovanni M, McInnes P, Wang L, Schloss JA, et al. The NIH Human Microbiome Project. Genome Res. 2009;19(12):2317-23. [PubMed: 19819907]

7. Wylie KM, Mihindukulasuriya KA, Zhou Y, Sodergren E, Storch GA, Weinstock GM. Metagenomic analysis of double-stranded DNA viruses in healthy adults. BMC Biol. 2014;12:71. [PubMed: 25212266]

8. Wylie KM, Wylie TN, Cahill AG, Macones GA, Tuuli MG, Stout MJ. The Vaginal Eukaryotic DNA Virome and Preterm Birth. Am J Obstet Gynecol. 2018;219(2):189.e1-.e12. [PubMed: 29738749]

9. Stout MJ, Zhou Y, Wylie KM, Tarr PI, Macones GA, Tuuli MG. Early pregnancy vaginal microbiome trends and preterm birth. Am J Obstet Gynecol. 2017;217(3):356.e1-.e18. [PubMed: 28549981]

10. Brook N, Khalaf Y, Coomarasamy A, Edgeworth J, Braude P. A randomised controlled trial of prophylactic antibiotics (co-amoxiclav) prior to embryo transfer. Hum Reprod. 2006;21(11):29115. [PubMed: 16832124]

11. Kaye L, Bartels C, Bartolucci A, Engmann L, Nulsen J, Benadiva C. Old habits die hard: retrospective analysis of outcomes with use of corticosteroids and antibiotics before embryo transfer. Fertil Steril. 2017;107(6):1336-40. [PubMed: 28501367]

12. Kroon B, Hart RJ, Wong BM, Ford E, Yazdani A. Antibiotics prior to embryo transfer in ART. Cochrane Database Syst Rev. 2012(3):CD008995. [PubMed: 22419341]

13. Practice Committee of the American Society for Reproductive Medicine. Performing the embryo transfer: a guideline. Fertil Steril. 2017;107(4):882-96. [PubMed: 28366416]

14. Gopinath S, Kim MV, Rakib T, Wong PW, van Zandt M, Barry NA, et al. Topical application of aminoglycoside antibiotics enhances host resistance to viral infections in a microbiota-independent manner. Nat Microbiol. 2018;3(5):611-21. [PubMed: 29632368]

15. Retallack H, Di Lullo E, Arias C, Knopp KA, Laurie MT, Sandoval-Espinosa C, et al. Zika virus cell tropism in the developing human brain and inhibition by azithromycin. Proc Natl Acad Sci U S A. 2016;113(50):14408-13. [PubMed: 27911847]

16. Beigelman A, Isaacson-Schmid M, Sajol G, Baty J, Rodriguez OM, Leege E, et al. Randomised trial to evaluate azithromycin's effects on serum and upper airway IL-8 levels and recurrent wheezing in infants with respiratory syncytial virus bronchiolitis. J Allergy Clin Immunol. 2015;135(5):1171-8 e1. [PubMed: 25458910]

17. Woods Acevedo MA, Erickson AK, Pfeiffer JK. The Antibiotic Neomycin Enhances Coxsackievirus Plaque Formation. mSphere. 2019;4(1):e00632-18. [PubMed: 30787120]

18. Elm EV, Altman DG, Egger M, Pocock SJ, Gøtzsche PC, Vandenbroucke JP. The Strengthening the Reporting of Observational Studies in Epidemiology (STROBE) Statement: guidelines for reporting observational studies. Int J Surg. 2014;12(12):1495-9. [PubMed: 25046131]

19. Wylie TN, Wylie KM, Herter BN, Storch GA. Enhanced virome sequencing using targeted sequence capture. Genome Res. 2015;25(12):1910-20. [PubMed: 26395152]

20. Wylie KM, Wylie TN, Buller R, Herter B, Cannella MT, Storch GA. Detection of Viruses in Clinical Samples by Use of Metagenomic Sequencing and Targeted Sequence Capture. J Clin Microbiol. 2018;56(12):e01123-18. [PubMed: 30232133]

21. Cardenas I, Mor G, Aldo P, Lang SM, Stabach P, Sharp A, et al. Placental viral infection sensitizes to endotoxin-induced pre-term labor: a double hit hypothesis. Am J Reprod Immunol. 2011;65(2): 110-7. [PubMed: 20712808]

22. Ledger WJ, Blaser MJ. Are we using too many antibiotics during pregnancy? BJOG. 2013;120(12):1450-2. [PubMed: 24118809]

23. Hosny A, El-Khayat W, Kashef MT, Fakhry MN. Association between preterm labor and genitourinary tract infections caused by Trichomonas vaginalis, Mycoplasma hominis, Gramnegative bacilli, and coryneforms. J Chin Med Assoc. 2017;80(9):575-81. [PubMed: 28094234]

24. Koullali B, Oudijk MA, Nijman TA, Mol BW, Pajkrt E. Risk assessment and management to prevent preterm birth. Semin Fetal Neonatal Med. 2016;21(2):80-8. [PubMed: 26906339] 
25. Meis PJ, Goldenburg RL, Mercer B, Moawad A, Das A, McNellis D, et al. The preterm prediction study: significance of vaginal infections. Am J Obstet Gynecol. 1995;173:1231-5. [PubMed: 7485327]

26. Flenady V, Hawley G, Stock OM, Kenyon S, Badawi N. Prophylactic antibiotics for inhibiting preterm labour with intact membranes. Cochrane Database Syst Rev. 2013(12):CD000246. [PubMed: 24307518]

27. Klebanoff MA, Carey JC, Hauth JC, Hillier SL, Nugent RP, Thom EA, et al. Failure of metronidazole to prevent preterm delivery among pregnant women with asymptomatic trichomonas vaginalis infection. N Engl J Med. 2001;345(7):487-93. [PubMed: 11519502]

28. Zimmermann P, Ziesenitz VC, Curtis N, Ritz N. The Immunomodulatory Effects of Macrolides-A Systematic Review of the Underlying Mechanisms. Front Immunol. 2018;9:302. [PubMed: 29593707]

29. Weiss G, Goldsmith LT, Taylor RN, Bellet D, Taylor HS. Inflammation in reproductive disorders. Reprod Sci. 2009;16(2):216-29. [PubMed: 19208790] 

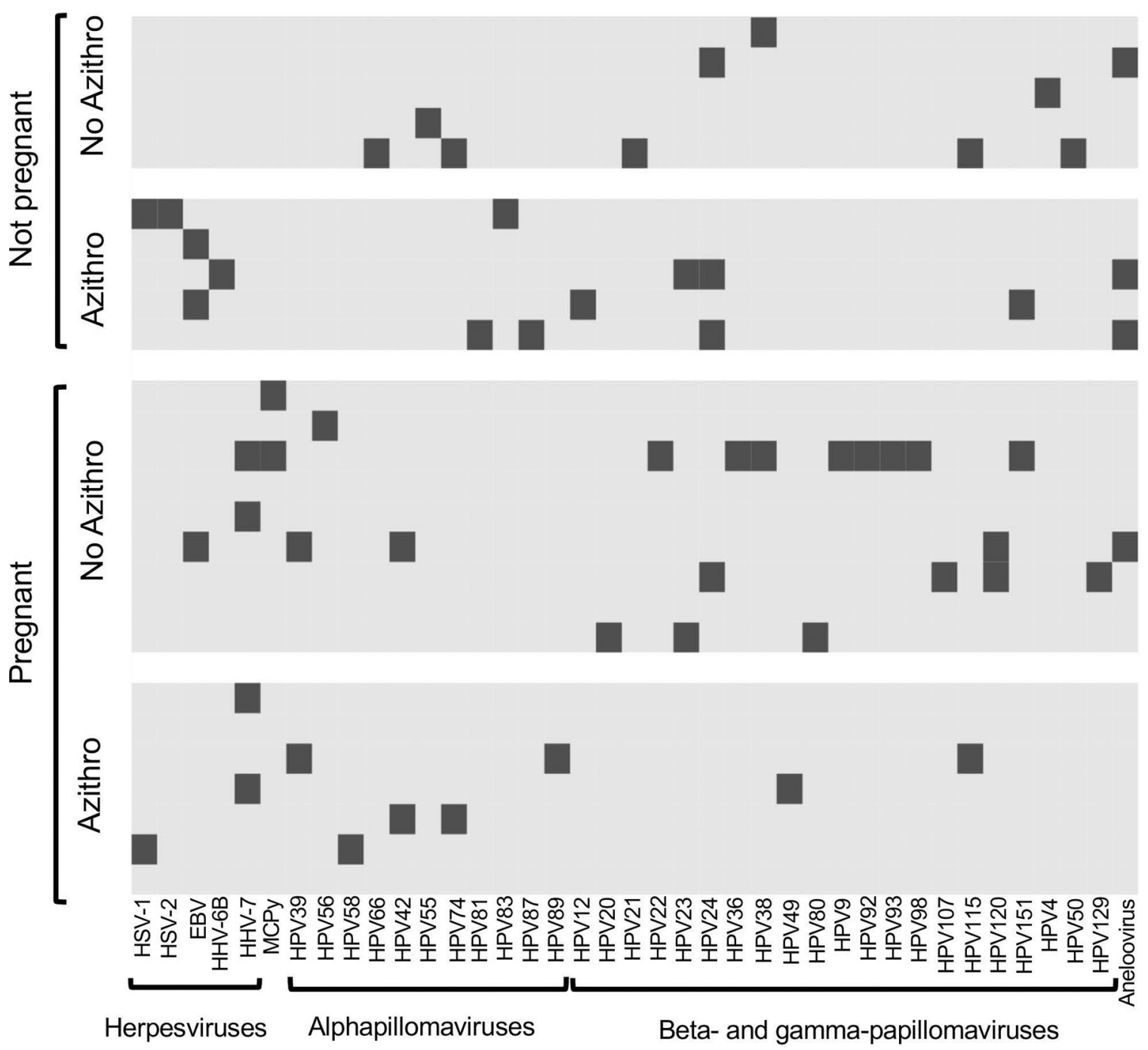

Figure1:

The vaginal virome in IVF patients at the time of embryo transfer. The viruses detected in each sample are shown. Each patient is represented in a row. Each virus detected is indicated in a column. The dark bars indicate the presence of the virus in the sample. Samples are grouped according to whether clinical pregnancy was achieved and according to whether they were included in the prophylactic azithromycin or no azithromycin groups of the study. HSV, herpes simplex virus. EBV, Epstein Barr virus. HHV, human herpes virus. MCPy, Merkel cell polyomavirus. HPV, human papillomavirus. 
A

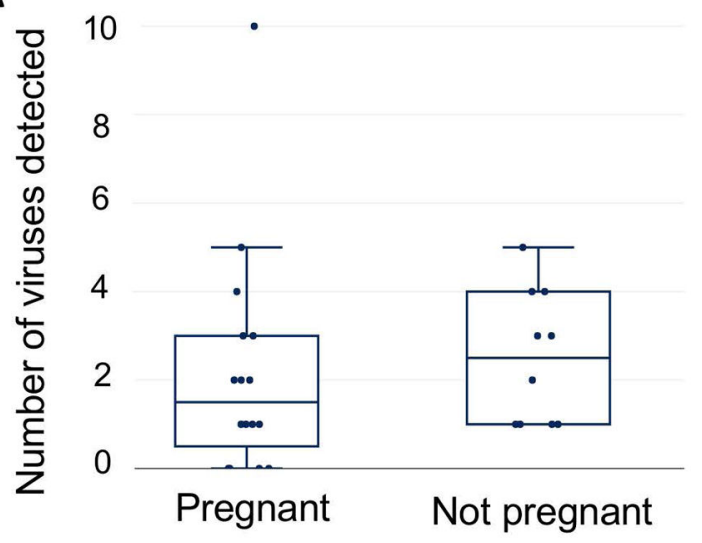

B

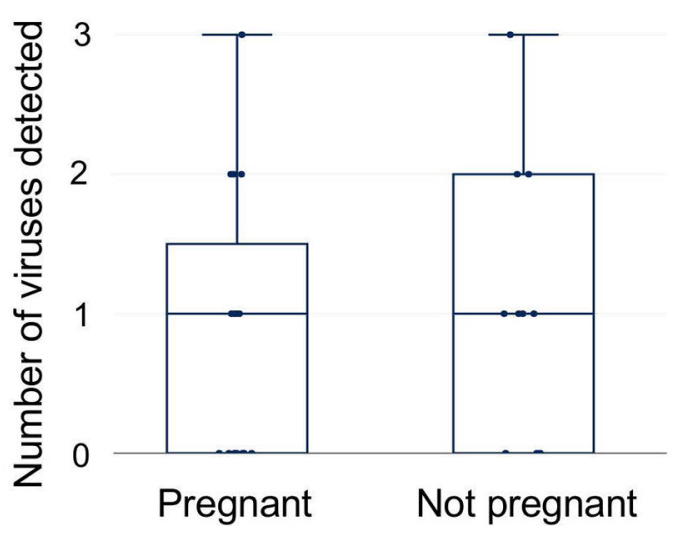

C

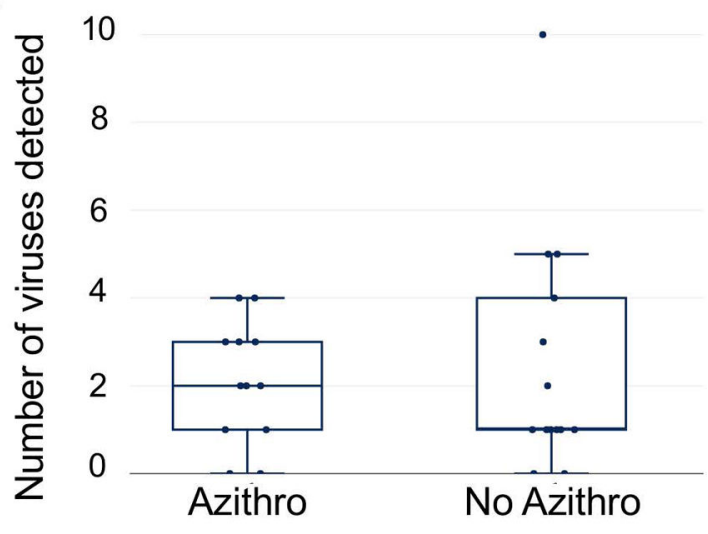

D

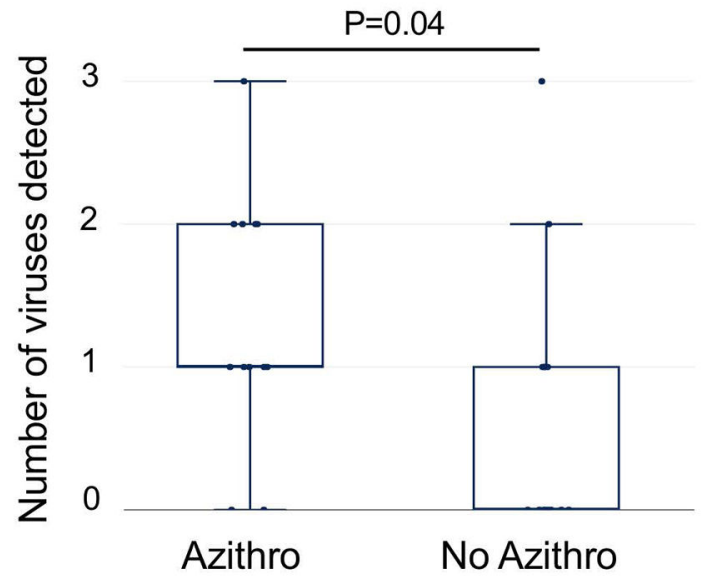

Figure2:

Associations between viral diversity with pregnancy or azithromycin prophylaxis. In panels A (all viruses) and B (herpesviruses and alphapapillomaviruses), viral diversity (total number of different viruses detected) in each sample are plotted using box and whisker plots, and pregnancy outcome are compared. The median is indicated by a horizontal line, the quartiles indicated by boxes, and minimum and maximum by the whiskers (outliers excluded). In panels $\mathrm{C}$ (all viruses) and $\mathrm{D}$ (the number of different alphapapillomaviruses and herpesviruses detected) are plotted using box and whisker plots, and azithromycin groups are compared. 

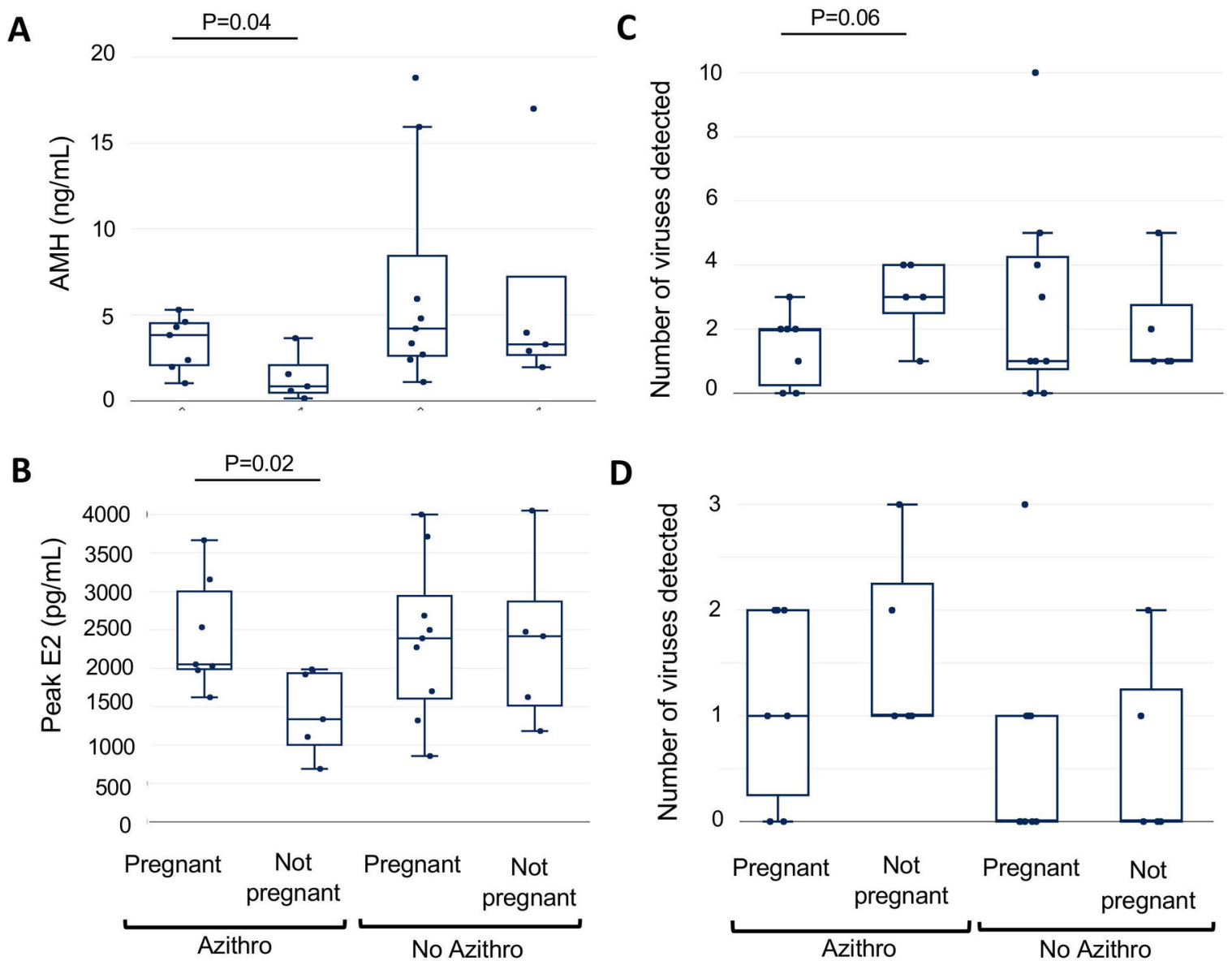

Figure3:

Associations between viral diversity and clinical characteristics with pregnancy and azithromycin prophylaxis. AMH levels (panel A), peak estradiol levels (panel B), viral diversity (the total number of viruses detected) (panel C), and number of alphapapillomaviruses and herpesviruses (panel D) are plotted using box and whisker plots, and groups are compared. 
Table 1-

Demographic and clinical characteristics of study participants.

\begin{tabular}{|c|c|c|c|}
\hline Variables & No Azithromycin (n=14) & Azithromycin $(n=12)$ & p-value \\
\hline Age (years) ${ }^{a}$ & $32.14(3.92)$ & $35.25(4.67)$ & 0.08 \\
\hline $\operatorname{BMI}\left(\mathrm{kg} / \mathrm{m}^{2}\right)^{a}$ & $31.53(8)$ & $30.48(7)$ & 0.73 \\
\hline Tobacco Use & 0 & 0 & - \\
\hline $\mathrm{AMH}(\mathrm{ng} / \mathrm{mL})^{a}$ & $2.29(1.06)$ & $1.47(0.62)$ & 0.03 \\
\hline $\mathrm{AFC}^{b}$ & $32(18,50)$ & $24(13,39)$ & 0.31 \\
\hline Race & & & 1.00 \\
\hline - White & $13(92.9 \%)$ & $12(100 \%)$ & \\
\hline - Asian & $1(7.1 \%)$ & $0(0 \%)$ & \\
\hline Ethnicity & & & 1.00 \\
\hline - Non-Hispanic & $14(100 \%)$ & $12(100 \%)$ & \\
\hline Duration of Infertility (months) $b$ & $25(18,48)$ & $18(8,32)$ & 0.15 \\
\hline Diagnosis & & & 0.20 \\
\hline - Male Factor & $5(35.7 \%)$ & $3(25 \%)$ & \\
\hline - Unexplained & $1(7.1 \%)$ & $4(33.3 \%)$ & \\
\hline - Tubal Factor & $2(14.3 \%)$ & $1(8.3 \%)$ & \\
\hline - Endometriosis & $1(7.1 \%)$ & $1(8.3 \%)$ & \\
\hline - Ovulatory Dysfunction & $1(7.1 \%)$ & $1(8.3 \%)$ & \\
\hline - PCOS & & & \\
\hline \multirow[t]{2}{*}{ - DOR } & $4(28.6 \%)$ & $0(0 \%)$ & \\
\hline & $0(0 \%)$ & $2(16.7 \%)$ & \\
\hline Protocol & & & 0.03 \\
\hline - Long Agonist & $9(64.3 \%)$ & $7(58.3 \%)$ & \\
\hline - Antagonist & $5(35.7 \%)$ & $1(8.3 \%)$ & \\
\hline - Flare & $0(0 \%)$ & $4(33.3 \%)$ & \\
\hline Prior Live Birth & & & 1.0 \\
\hline - Yes & $3(21.4 \%)$ & $2(16.7 \%)$ & \\
\hline - No & $11(78.6 \%)$ & $10(83.3 \%)$ & \\
\hline
\end{tabular}

BMI, body mass index.

$\mathrm{AMH}$, antimullerian hormone.

AFC, antral follicle count.

PCOS, polycystic ovarian syndrome.

DOR, diminished ovarian reserve.

${ }^{a}$ Mean \pm standard deviation

$b_{\text {Median (interquartile range) }}$ 